\title{
Machine intelligence approach for optimization of cranial tumor image
}

Tamsekar P.B. and Gomase V.S.

\author{
School of Technology, S.R.T.M. University, Sub-Centre, Latur, 413512, India
}

\begin{abstract}
High-throughput image analysis is very important aspect of modern post-genomics and proteomics research. Image optimization technology is the driving force of this revolution and technology, which allows the simultaneous monitoring of expression for thousands of images. The need for accurate and reproducible research has driven the development of robust analysis frameworks for maximizing the information content of cancer pathological data. Image optimization is a powerful tool has multiple applications both in clinical and cellular and molecular biology arenas. Image analysis technology has shown new advancements in the field of biomedical research and diagnosis, it allows studying and understanding tumor activities and interactions in malignancies or diseases; therefore, it has great potential for clinical diagnostics in the future.
\end{abstract}

Keywords- High-throughput analysis, optimization, cancer, SVM, MRF, CRF

\section{Introduction}

High-throughput image analysis is an important aspect of modern post-genomic research. Image optimization technology is the driving force of this revolution, a technology that allows the simultaneous monitoring of expression for thousands of images. The need for accurate and reproducible research has driven the development of robust analysis frameworks for maximizing the information content of pathological data. Image optimization is a powerful tool has multiple applications both in clinical and cellular and molecular biology arenas. Image Analysis technology has shown great advancements in the field of biomedical research and diagnosis, it allows studying and understanding tumor activities and interactions in malignancies or diseases; therefore, it has great potential for clinical diagnostics in the future. A set of tumor images are acquired by a sequence of biological experiments which were scanned via a high resolution scanner. For each spot corresponding to the ratio of fluorescent signal intensities is obtained and which may be normalized based on piecewise [1]. We focus on two important issues; the first issue is related to automatic gridding and spot segmentation for tumor images. It has been reported that the quality of spot segmentation significantly influences data precision in the subsequent data analysis. Also, now a days tumor image analysis software still requires users' fine tuning to obtain acceptable results. Another important issue is how to automatically collect related information regarding all images on the tumor slide for subsequent data analysis and data mining. To relieve researchers from manually correcting image processing results and manually collecting the related information for tumor images, in these techniques, we proposed an automatic and robust method for tumor image analysis and the related information retrieval module which is integrated with the proposed database schema for brain tumor image data. Algorithm is presented which aims at the deflective problem of brain tumor image. This method is based on image power spectra [2]. Examined by hundreds of samples of clinical data, the algorithm is proved to achieve high precision. As a result, adopting this algorithm, the overall procedure automation in tumor image analysis can be realized.

\section{Origin of the proposal}

The brain tumor image optimization technique is assuming even more importance. Digital images acquisition becomes fundamental to provide an automatic system for subsequent analysis. Image segmentation has been researched for a long time from early vision but it is yet a challenging task now. Many segmentation methods can be classified roughly into three groups, local method, area-based method, and global optimization method [3]. The local method relies on local information in an image for finding contours and edges of regions. It suffers from grouping edge fragments and differentiating region edges from texture edges. The area-based method used region growing or splitting/merging techniques. However, it is often confronted with difficulties in detecting reasonable region boundaries [4]. The global optimization method partitions an image into disjoint regions fulfilling some homogeneity criteria. However, the homogeneity criteria require restrictive texture and object models to overcome non-uniformity of colors and textures in a region due to distance, foreshortening, shading, and illumination. Therefore, the global optimization method usually requires expensive computation.

\section{Definition of the problem}

Image optimization technology is a powerful tool for analyzing the expression of a large number of tumor affected images in parallel. A typical brain tumor image consists of a few thousands of spots which determine the level of cancer expression in the sample. Initially, a preliminary segmentation of the image is produced using a template matching algorithm. Next, grid and spot finding are realized. The position of non-expressed spots 
is located and finally an employ to fit the grid on the image [5]. A rough and fast segmentation method of color images is proposed which uses dissimilarity of local color distribution to measure discontinuity of colors and textures. The dissimilarity of local color distribution is computed using a modified color histogram intersection technique that enables us to measure the discontinuity of colors at boundaries of objects efficiently and to lessen the effect of discontinuity at finely textured regions [6, 7]. The watershed transformation technique is used to find. A rough and fast segmentation method of color images is proposed, which used dissimilarity of color distribution to measure discontinuity of colors and textures. Through several experiments, the proposed dissimilarity will useful for representing the discontinuity of colors at boundaries of objects and lessening the effect of discontinuity at boundaries of textures. The proposed merging method based on the watershed transformation also shows the most important salient regions of images could be segmented fast and well even though their boundaries were a little rough [8]. The proposed segmentation method is expected to be useful for effective content-based image retrieval at the level of objects from tumor $[9,10]$.

\section{Context of current status}

This work presents cancer laboratory application, where a combined texture and color analysis technique is used for the classification of industrial products. Color and texture have been largely studied and many methods have been proposed in order to handle machine vision problems where color and texture features serve as a cue for classification, segmentation and recognition. The obtained results are promising and show the possibility of efficiently classifying complex cancer laboratory products based on color and texture features.

\section{Technological utility}

The aim of the work is to provide pathologist community a simplified tool to manage their experiments in order to facilitate data exchange and integration with other information available on line. The platform arises from the necessity, not solved by other existing platforms, to enrich the support given to brain tumor images optimization experiments, because in our opinion the communication between pathologists, scientists and bioinformaticians of different institutes is important for the spreading of this technique. Indeed, a peculiarity of the proposed system is the integration in the application of a strong ontology mechanism to guarantee standardized descriptions for computational methods and experiment outputs. Moreover, the system provides high integration to molecular biology knowledge: although the technique is pretty close to the bioinformatics field, there are no references in other platforms about managing brain tumor images data coming from brain tumor optimization experiments. Method is compared to widely used, state-of-the-art segmentation methods in brain tumor image analysis in a study of a metabolic disorder, where replicates of reporters are present. Method yields more reproducible log ratio measurements across replicates.

\section{Methodology for semantic approach}

Digital images acquisition becomes fundamental to provide an automatic system for subsequent analysis. The accuracy of the results depends on the image resolution, which has to be very high in order to provide as many details as possible. Lossless formats are more suitable to bring information, but data file sizes become a critical factor researchers have to deal with. This affects not only storage methods but also computing times and performances. Pathologists and researchers, who work with biological tissues, in particular with the brain tumor image analysis technique, need to consider a large number of case studies to formulate and validate their hypotheses [12-14]. It is clear the importance of image sharing between different institutes worldwide to increase the amount of interesting data to work with. Various statistical descriptors will proposed for the measure of image textures. Theses statistical approaches use n-order statistics to define image textures. Other approaches define textures by means of mathematical morphology operators or Filter Banks. Many of these techniques are first propose for processing images, and then were extended for color texture processing. Proposed the use of a combination of color and texture features; texture features are computed in color scale and combined with color histograms and moments. These combined features are then sent to a classifier for color-texture classification. Other proposed the use of color quantization to reduce the number of colors and process the resulting image as for texture extraction. More sophisticated techniques use a combination in between color bands for texture feature computation [15-17]. For color-texture classification we propose an approach based on a generalization of matrix to color images. For comparing two color images, we extract the three color bands for each image and compute the matrix. Texture features are computed from the matrix and combined with color band entropy. These measures are compared to the corresponding features in the second image. The obtained distance is a measure of similarity between the two images. If this distance is below a user specified threshold then the two images are classified as similar, otherwise the two images are classified as non-similar [18-21]. This scheme has the advantage of effectively 
encoding complex non homogenous textures where irregular components are present and textures lacking repetitive patterns. These textures can be characterized by the spatial cooccurrence of their color components making the matrix a suitable method for our analysis. The proposed method confirms that the self-learning ability and adaptability of the self-organizing map, coupled with the information fusion mechanism of the hierarchical network, leads to superior segmentation results for Brain Tumor images [2225]. One of the major factors that complicate the task of brain tumor image analysis is that brain tumor images are distorted by various types of noise. In this study a robust framework is proposed, designed to take into account the effect of noise in brain tumor images in order to assist the demanding task of brain tumor image analysis [26, 27]. The proposed framework incorporates in the brain tumor image processing pipeline a novel combination of spot adjustable image analysis and processing techniques and consists of the following stages:

1. Gridding for facilitating spot identification,

2. Clustering (unsupervised discrimination between spot and background pixels) applied to spot image for automatic local noise assessment,

3. Modeling of local image restoration process for spot image conditioning (adjustable wiener restoration using an empirically determined degradation function),

4. Automatic spot segmentation employing seeded-region-growing,

5. Intensity extraction and

6. Assessment of the reproducibility (real data) and the validity (simulated data) of the extracted gene expression levels.

\section{Model Classification}

$\mathrm{MRI}$ is considered the ideal method for brain imaging. The 3D data and the large number of possible protocols allow identifying anatomical structures as well as abnormal brain structures.

\section{Supervised and Un-Supervised Segmentation Methods}

Supervised and un-supervised methods for image processing are frequently applied $[73,71$, 72] Supervised classification enables us to have sufficient known pixels to generate representative parameters for each class of interest. In an unsupervised classification pre hand knowledge of classes is not required and usually employees some clustering algorithm for classifying an image data. According to [71] KNN, ML and Parzen window classifiers are supervised classification algorithm. Whereas, un-supervised classification algorithm includes: KMeans, minimum distance, maximum distance and hierarchical clustering etc. Alternatively, different machine learning (ML) classification techniques have been investigated: SVMs (Support Vector Machines), MRFs(Markov Random Fields) and most recently CRFs (Conditional Random Fields) [51].

\section{SVMs (Support Vector Machines)}

We extract a candidate set of segments from the intermediate level of the pyramid (scales 5,6 from all 13 scales) which correspond to brain tissue regions. To construct the classifier we utilize "ground-truth" expert segmentation which is provided along with the real clinical brain MRI data. Generally we assume (1) having a training sample of $M$ candidate segments, Cand $=\{f 1$. . $\mathrm{fM}$, each described by a d-dimensional feature vector (we normalize each of the features to have zero mean and unit variance), and (2) a mask indicating the voxels marked by an expert as GM, WM, CSF and background. Since many of the segments may contain a mixed collection of the categories, the labeling category is determined based on the category marked by the maximum number of voxels associated with the segment [60]. For classification we apply automatic learning, procedure based on an SVM algorithm using data pre-labeled by experts. In the case of brain tumor segmentation, the lack of shape or intensity priors on the tumors, makes it challenging to proceed in an unsupervised manner. Some recent progress has been made to create semi-supervised [64] (based on user interaction) or supervised variational methods [65].

\section{MRFs (Markov Random Fields) and CRFs (Conditional Random Fields)}

Researchers are apply Markov Random Fields (MRFs; [66]) and Conditional Random Fields (CRFs; [67]) to various segmentation tasks. These techniques are able to represent complex dependencies among data instances, giving them higher accuracy on the segmentation task tshan iid classifiers [67, 68]. However, these random field approaches are based on computationally intractable formulations. Although there are approximation techniques that can deal with these computational challenges, CRF variants such as Discriminative Random Fields (DRFs) and Support Vector Random Fields (SVRFs) still require computationally expensive learning procedures $[67,69]$. Pseudo-Conditional Random Fields (PCRFs) achieve accuracy similar to other random fields variants but are significantly more efficient. PCRF regularize discriminative classifier that relaxes the classification decision for each voxel by considering the labels and features of neighboring voxels $[65,70]$.

\section{Separating tumor and ventricles using a Dirichlet prior \\ Most variational segmentation methods are used} in an unsupervised setting where the region 
statistics are refined as the curve evolves. This might be quite effective if the region statistics are distinct [58]. But, as mentioned earlier, one of the main problems in brain tumor segmentation is that the appearance of tumor and surrounding tissue are not always clearly separated (not even in the feature space).We therefore have to use additional prior information to help the segmentation. The tumor doesn't have a particular shape prior. In addition, the surrounding tissues (like the ventricles) can be deformed and therefore don't preserve a shape prior. We chose to use a prior on the appearance that better disambiguate the two regions [58-59].

\section{Conclusion}

Existing region-based variational segmentation Methods based on texture features are not suited for tumor segmentation as they are not discriminative enough when the appearance of tumor and normal tissue overlap. PCRF (SVM) system, which uses a linear SVM to map from voxel to label, worked effectively.

\section{References}

[1] Mahadevan-Jansen A. (2007) Optical Guidance of Brain Therapies, 1- 2.

[2] Suzuki M., Kato K., Hirashima T., Shindo Y., Uzuka T., Takahashi H.,

Fujii Y. (2009) Annual International Conference of the IEEE ,3064-3067.

[3] Shindo Y., Kato K., Tsuchiya K., Yabuhara T., Shigihara T., Iwazaki R., Uzuka T., Takahashi H., Fujii Y. (2007) $29^{\text {th }}$ Annual International Conference of the IEEE-3609.

[4] Mohri K., Shinkai M., Ohshima A., Honda H., Kobayashi T., Wakabayashi T., Yoshida J. (1997) IEEE Transactions, 33(5), Part 2,4266-4268.

[5] Ravi Doraiswami, Kalapurakal J.A. (2006) Electronic Components and Technology Conference, 4.

[6] Corso J.J., Sharon E., Dube S., El-Saden S., Sinha U., Yuille A. (2008) IEEE Transactions, 27 (5), 629-640.

[7] Yun Zhou, Huang S.C., Cloughesy T., Hoh C.K., Black K., Phelps M.E. (1997) IEEE Transactions, 44(6), Part 2, 2522-2526.

[8] Zhou J., Chan K.L., Chong V.F.H., Krishnan S.M. (2005) IEEE-EMBS, 27th Annual International Conference of the 2006, 64116414.

[9] Wang C.H., Tseng S.S. (1990) IEEE Symposium, 313-320.

[10] Yun Zhou, Sung-Cheng Huang, Shanglian Bao, Wong D.F. (2001) Nuclear Science Symposium Conference Record, IEEE, 2, 1072-1078.

[11] Shen S., Sandham W.A., Granat M.H., Dempsey M.F., Patterson J., (2003) Proceedings of the 25th Annual International Conference of the IEEE, 1, 870-873.
[12] Lin J.C. (2009) Microwave Magazine, IEEE, 10(4), 138, 140-140,144.

[13] Zacharaki E.I., Sumei Wang, Chawla S., Dong Soo Yoo, Wolf R., Melhem E.R., Davatzikos C., (2009) IEEE International Symposium, 1035-1038.

[14] Yabuhara T., Kato K., Kanazawa Y., Kubo M., Takahashi H., Uzuka T., Fujii Y., (2008) Annual International Conference of the IEEE, 20-25, 4364-4367.

[15] Salman Y.M., Assal M.A., Badawi A.M., Alian S.M., El-Bayome, M.El.; (2005) IEEE, 27th Annual International Conference, 70487051.

[16] Tao Wang, Cheng I., Basu A. (2009) IEEE Transactions, 56(3), 781- 789.

[17] Dhouib D.,Nait-Ali A., Olivier C., Naceur M.S. (2009) $6^{\text {th }}$ International Multi-Conference, 23-26, 1 - 6.

[18] Saxena V., Nielsen J.F., Gonzalez-Gomez I., Karapetyan G., Khankaldyyan V., Nelson M.D., Laug W.E. (2005) Applied Imagery and Pattern Recognition Workshop, 6-132.

[19] Boukerche A., Maamar H., Hossain A., (2007) Computers and Communications, ISCC. 12th IEEE Symposium 975-980.

[20] Vinitski S., Gonzalez C., Burnett C., Buchheit W., Mohamed F., Ortega H., Faro S. (1995) IEEE 17th Annual Conference,1, 481-482.

[21] Elamy A.H., Maidong Hu, (2007) Electrical and Computer Engineering, CCECE, 872875.

[22]Chunyan Jiang, Xinhua Zhang, Wanjun Huang, Meinel C. (2004) IEEE Symposium, 61-66.

[23] Dellani P.R., Wille P.R., Vucurevic G., Bauermann T., Stoeter P., Glaser M., Stadie A., Grunett P., Perneczky A., von Wangenheim A. (2004) 17th IEEE Symposium, 367-372.

[24] Glotsos D., Spyridonos P., Petalas P., Cavouras D., Zolota V., Dadioti P., Lekka I., Nikiforidis G. (2003) International Symposium, 2,1034-1038.

[25] Leung C.C., Chen W.F., Kwok P.C.K., Chan F.H.Y. (2003) International Conference, 2, 1057-60.

[26] Stupak V.V., Ogirenko A.P., Denisov A.N., Nikonov C.D., Pendyurin I.V., Zaidman A.M. Serpeninova N.N. (2000) 5th International Conference, 202-204.

[27] Zacharaki E.I. Hogea C.S., Biros G., Davatzikos C. (2008) IEEE, 55(3), 12331236.

[28] Kounelakis M. G., Zervakis M. E., Postma G. J., Buydens L. M. C., Heerschap A., Kotsiakis X. (2009) Annual International Conference of the IEEE, 35-38.

[29] Mimoto N., Kato K., Kanazawa Y., Shindo Y., Tsuchiya K., Kubo M., Uzuka T., Takahashi H., Fujii Y. (2009) Annual 
International Conference of the IEEE, 3068-3071.

[30] Das A., Bhattacharya M., (2009) IJCBS '09. International Joint Conference, 348-351.

[31] Hemanth D.J., Selvathi D., Anitha J., (2009) Advance Computing Conference, 609-614.

[32] Bourouis S., Hamrouni K., (2008), 1-5.

[33] Farias G., Santos M., Lopez V. (2008) International Conference, 1, 1453-1459.

[34] Kounelakis M.G., Zervakis M.E., Blazadonakis M.E., Postma G.J., Buydens L.M.C., Heerschap A., Kotsiakis X. (2008) IEEE International Conference, 1-6.

[35] Poullet J.-B., Martinez-Bisbal, M.C., Valverde D., Monleon D., Celda B., Arus C., Van Huffel S. (2007) International Conference of the IEEE 5407-5410.

[36] Iftekharuddin K.M., Zheng J., Islam M.A., Ogg R.J., Lanningham F., (2006), Fortieth Asilomar Conference, 1983-1987.

[37] Devos A., Lukas L., Simonetti A.W., Suykens J.A.K., Vanhamme L. van der Graaf M., Buydens L.M.C., Heerschap A., Van Huffel S., (2004) IEEE, 1, 407-410.

[38] Cosic I., Pirogova E., Akay M., (2003) 25th Annual International Conference of the IEEE 3, 2398-2401.

[39] Dahiya S., Chaudhury K., Singh V.R., (1997) 19th Annual International Conference of the IEEE, 2, 832-834.

[40] Paglione R.W., Sterzer F., Winter A., Laing J., (1986) Microwave Symposium Digest, 86(1), 767-769.

[41] Schmidt M., Levner I., Greiner R., Murtha A., Bistritz A. (2005) International Conference, 6 .

[42] Zhou Y., Huang S.C., Cloughesy T., Hoh C.K., Black K., (1996) IEEE, 3, 1859-1862.

[43] Drake J.M., Joy M., Goldenberg A., Kreindler D. (1991) 'Robots in Unstructured Environments', ICAR, International Conference, 888- 892.

[44] Lin J.C. (2009) IEEE, 51(1), 182-184.

[45] Arndt Jovin D.J., Kantelhardt S.R., Caarls W., de Vries A.H.B., Giese A., Jovin T.M. (2009) NanoBioscience, IEEE, 8(1), 65-71.

[46] Zacharaki E. I., Shen D., Lee S.K., Davatzikos C., (2008) IEEE, 27(8), 10031017.

[47] Dong yong Dai, Condon B., Hadley D., Rampling R., Teasdale G. (1993) IEEE, 12(4), 693-702.

[48] Lin J.C., Shinji Hirai, Chin-Lin Chiang, WenLin Hsu, Jenn-Lung Su, Yu-Jin Wang (2000) IEEE Transactions, 48, 2191-2198.

[49] Lin J.C., (2001) IEEE, 2(4), 26-30.

[50] Predd P.P., (2004) IEEE, 41(12), 20-22.

[51] Karteek Popuria, Dana Cobzasb, Martin Jagersandb, Sirish L. Shaha and Albert Murthac , 1-2.

[53] Gibbs P., Buckley D., Blackb S. and Horsman A. (1996) 41, 2437-2446.
[54] Schmidt M., (2005) Master's thesis, Univ. of Alberta.

[55] Gering D., (2003) PhD thesis, MIT.

[56] Lee C.H., Schmidt M., Murtha A., Biaritz A., Sander J. and Greiner R. (2005) Workshop on CVBIA at ICCV.

[57] Corso J. J., Sharon E., Dube S., El-Saden S., Sinha U. and Yuille A., Medical Imaging, (2008) IEEE, 629-640.

[58] Chan T. and Vese L. (2001) IEEE, 10(2), 266-277.

[59] Rousson M., Brox T. and Deriche R. (2003) [CVPR].

[60] Vapnik V., (1995) Springer-Verlag.

[61] Akselrod Ballin A., Galun M., Gomori J.M., Fillipi M., Valsasina P., Brandt A., R.Basri (2006) CVPR.

[62] Zijdenbos A., Dawant B. (1994) Critical Reviews in Biomedical Engineering.

[63] Gerig G., Jomier M., Chakos M. Valmet (2001) MICCAI, 516-523.

[64] Cremers D., Fluck O., Rousson M. and Aharon S. (2007) [Proc. of the SPIE Med. Imaging].

[65]Cobzas D., Birkbeck N., Schmidt M. and Jagersand M. (2007) [MMBIA in conjunction with ICCV].

[66] Li S.Z. (2001) Springer-Verlag, Tokyo

[67] Lafferty J., Pereira F., McCallum A. (2001) $I C M L$

[67] Lee C.H., Greiner R., Schmidt M. (2005) PKDD, 121-132.

[68] Lee C.H., Wang S., Jiao F., Schuurmans D., Greiner R. (2007) NIPS 19.

[69] Kumar S., Heber M. (2003) NIPS.

[70] Ng A., Jordan M. (2002) NIPS 14.

[71] Guillermo N. Abras and Virginia L. Ballarin, (2005) JCS\&T, 5, 3.

[72] J.C. Bezdek, L.O. Hall, L. P. Clarke (1993) Medical Physics, 20, 4.

[73] Matthew C. Clark (1994) MS Thesis, Department of Computer Science and Engineering, University of South Florida. 